\title{
Effect of low-level laser therapy on tissue repair after dental extraction in rats administered zoledronic acid and dexamethasone
}

João Batista Blessmann Weber

Renata Stifelman Camilotti

Juliana Jasper

Liliane Cristina Onofre Casagrande

Fábio Luiz Dal Moro Maito 


\title{
Effect of low-level laser therapy on tissue repair after dental extraction in rats administered zoledronic acid and dexamethasone
}

\author{
João Batista Blessmann Weber, ${ }^{*}$ Renata Stifelman Camilotti, Juliana Jasper, \\ Liliane Cristina Onofre Casagrande, and Fábio Luiz Dal Moro Maito \\ Pontifícia Universidade Católica do Rio Grande do Sul (PUCRS), Porto Alegre, Brazil
}

\begin{abstract}
Bisphosphonates (BPs) are being increasingly used for the treatment of metabolic and oncological pathologies involving the skeletal system. Because of the severity of the BP associated osteonecrosis of the jaws, the difficulties of treatment, and patient discomfort, additional support methods for their management are needed. Laser therapy has an easy handling, photobiostimulator effect on tissues healing, so it can be considered a preferred therapy. The aim of this study was to evaluate the influence of low-level laser therapy in the 685and 830-nm wavelength in the healing process of the bone and soft tissues in rats under BP therapy [zoledronic acid (ZA)] and dexamethasone concomitantly that underwent a surgery for the extraction of upper molars. There were statistically significant differences in the clinical evaluation of the wound and the weight of the animals. Regarding the histological evaluation, it was possible to observe the different maturations of the healing stage between groups. The effect of drug therapy with ZA and dexamethasone in the bone tissue repair process induces osteonecrosis of the jaw in rats and slows down the healing process. In the laser groups, at the stipulated dosimetry, a positive influence on the bone and soft tissue repair process was observed. @2017 Society of Photo-Optical Instrumentation Engineers (SPIE) [DOI: 10.1117/1.JBO.22.5.058001]
\end{abstract}

Keywords: low-level light therapy; lasers; bisphosphonate; bisphosphonate related osteonecrosis of the jaw; animal experimentation. Paper 170061R received Jan. 24, 2017; accepted for publication Apr. 17, 2017; published online May 13, 2017.

\section{Introduction}

Bisphosphonates (BPs) are a class of drugs widely used in the treatment of metabolic diseases and malignancies that affect the skeletal system, including osteoporosis, Paget's disease of bone, and bone metastases from malignant neoplasms (such as multiple myeloma, breast cancer, or prostate cancer). ${ }^{1-5}$ BPs constitute a single class of medications, divided into firstand second-generation agents, which have been shown to inhibit osteoclasts and, possibly, interfere with angiogenesis through several mechanisms of action, including inhibition of vascular endothelial growth factor. ${ }^{6}$ The efficacy of BPs is attributed to their highly specific affinity for bone and their minimal biotransformation. While BPs have positive effects on bone diseases, the second-generation agents (those BPs that contain nitrogen atoms in their chemical structures) can induce necrosis of the jaw bones. This adverse effect, known as bisphosphonaterelated osteonecrosis of the jaw (BRONJ), is a result of reduced bone resorption and remodeling. ${ }^{5-9}$ BRONJ is characterized mainly by painful oral ulcers. Patients are also prone to nonhealing bone necrosis, which may affect the maxilla, the mandible, or both; however, due to its predominantly cortical structure, the mandible is more commonly involved, and these lesions are particularly difficult to treat. ${ }^{2,10-12}$ The risk of bone necrosis is substantially higher in patients receiving intravenous BPs. ${ }^{11}$ Signs and symptoms such as edema, dental mobility, sinusitis, oral or cutaneous fistulas, and pathological fracture of the mandible are commonly related to BRONJ. These symptoms

*Address all correspondence to: João Batista Blessmann Weber, E-mail: jbbweber@terra.com.br adversely affect quality of life and are a cause of significant morbidity in patients with this condition. ${ }^{13}$ Histopathologically, BRONJ lesions are usually characterized by necrotic bone, with loss of the lamellar bone meshwork, empty lacunae, absence of haversian canals, effacement of blood vessels, and presence of a mixed lymphocyte/plasma-cell infiltrate. Microbial pathogens, including Candida albicans or Actinomyces, may be present. ${ }^{13}$ The trigger events most commonly reported as causes of BRONJ are dental extraction, bone surgery, periodontal disease, and dental prosthesis-related trauma, although spontaneous cases have also been reported. ${ }^{14,15}$ In 2014 , a special committee of the American Association of Oral and Maxillofacial Surgeons recommended that BRONJ be renamed medication-related osteonecrosis of the jaw. This change was justified by the rising number of cases of osteonecrosis of the maxilla and mandible associated with other, non-BP antiresorptive and antiangiogenic therapies. $^{16}$

Given the severity of BRONJ, the challenges of treatment, and the discomfort experienced by patients, additional methods for its management are urgently needed. Laser-based therapy, which is easy to use and has demonstrated beneficial effects on bone healing, may be an important adjunct in treatment protocols. ${ }^{5}$ Through photobiomodulation of cell metabolism, laser therapy can improve wound healing and relieve pain, which makes it a potentially promising modality for the treatment of BRONJ. ${ }^{2,17}$ The photostimulation promoted by laser energy appears to activate the lymphatic system; has effects on macrophage, lymphocyte, endothelial cell, epithelial cell, keratinocyte, and fibroblast proliferation; and increases production of

1083-3668/2017/\$25.00 @ 2017 SPIE 
mitochondrial enzymes, porphyrins, flavins, and cytochromes. ${ }^{1,17}$ The effects of the laser on nutrition of the skin and mucous membranes, both in vivo and in vitro, have been reported by many authors and may provide evidence for the use of laser biostimulation in the treatment of BRONJ. ${ }^{10}$

Most reported cases of BRONJ have occurred in patients under treatment for multiple myeloma or breast cancer. ${ }^{14}$ These patients receive nitrogen-containing bisphosphonates, including zoledronic acid (ZA) and pamidronate, via the intravenous route. ${ }^{1,10,14,18}$ In addition to route of administration, other risk factors have also been noted to influence BRONJ development. Few studies have assessed the effects of a laser on healing in BRONJ, and conducting such research in humans may be difficult. For the aforementioned reasons, this study was conducted in animals, as were previous experiments. ${ }^{17-22}$

The objective of this study was to evaluate the effects of lowlevel laser therapy (LLLT) in the red and infrared wavelengths on bone and soft-tissue repair in rats with experimental BRONJ induced by combination BP (ZA) and glucocorticoid [dexamethasone (DX)] therapy and subjected to surgical extraction of upper molars. The combination of ZA and DX was designed to reproduce regimens administered to humans with multiple myeloma, which are among the therapies most associated with BRONJ. $^{18}$

\section{Materials and Methods}

\subsection{Animals}

This study was approved by the relevant institutional Animal Care and Use Committee (CEUA PUCRS) and was conducted in accordance with the Animal Research: Reporting of In Vivo Experiments (ARRIVE) guidelines. The study sample consisted of clinically healthy, heterogeneous male Wistar rats (Rattus norvegicus albinus), with a mean age 8 weeks and a mean weight $225 \pm 25 \mathrm{~g}$. Animals were housed in plastic boxes lined with autoclaved natural wood bedding. One animal from each group was allocated to each box, for a total of four animals per box. These boxes were placed on ventilated cage racks (Tecniplast, Buguggiate, Varese, Italy) in a facility with controlled temperature $\left(24^{\circ} \mathrm{C} \pm 2^{\circ} \mathrm{C}\right)$ and humidity $(50 \% \pm 5 \%)$. Animals were kept under a 12-h light-dark cycle, fed standard laboratory chow (Nuvilab, Colombo, Paraná, Brazil), and given free access to filtered drinking water. To prevent behavioral stress and other immune or metabolic alterations, no experimental procedures were performed in the animal housing facility.

\subsection{Study Design}

Twenty-eight rats were randomly divided into four groups of seven animals each: one vehicle control group, one medication control group (administered ZA and DX), and two experimental groups, which were administered ZA and DX and treated with LLLT. All animals underwent extraction of the left maxillary first and second molars. Extractions were performed 36 days after the beginning of the medication regimen, which consisted of 35 days of DX (administered subcutaneously at $1 \mathrm{mg} / \mathrm{kg}$ once daily) and five doses of ZA (administered subcutaneously at $7.5 \mathrm{mg} / \mathrm{kg}$ every 7 days for 5 weeks). Animals in the vehicle control group were administered normal saline solution $(\mathrm{NaCl}$ $0.9 \%$ ) at the same volumes used in the medication groups. Animals were weighed once weekly, starting on the first day of the experiment.
All animals were euthanized 14 days after dental extractions. The left maxilla of each animal was evaluated clinically and photographs were obtained. The maxillae were then dissected out, divided longitudinally with the surrounding soft tissues, fixed in $10 \%$ formalin for at least $72 \mathrm{~h}$, and decalcified in nitric acid for 36 to $48 \mathrm{~h}$. The areas of interest were then cut from these specimens and stored in nitric acid for a further 24 to $36 \mathrm{~h}$. The resulting specimens were then paraffin-embedded and sliced into $5-\mu \mathrm{m}$ sections, which were stained with hematoxylin and eosin (H\&E).

\subsection{Study Procedures}

\subsubsection{Dental extractions and postoperative care}

Before surgery, each rat was weighed to calculate the anesthetic dose. General anesthesia was induced by intraperitoneal injection of a mixture of $20 \mathrm{mg} / \mathrm{mL}$ of xylazine hydrochloride (0.05 mL/100 g weight; Anasedan, Ceva Saúde Animal Ltda, Paulínia, São Paulo, Brazil), sedative, analgesic, and muscle relaxant with $100 \mathrm{mg} / \mathrm{mL}$ of ketamine hydrochloride (0.1 mL/100 g weight; Dopalen, Ceva Saúde Animal Ltda, Paulínia, SP, Brazil), an anesthetic for veterinary use.

All left maxillary first- and second-molar extractions were performed by an experienced oral and maxillofacial surgeon. Briefly, after separation from the surrounding gum tissue and severing of the periodontal ligament, teeth were luxated with a periotome and extracted with a curved hemostat. Occasionally, dental fractures occurred. Neither alveoloplasty nor septal alveolectomy were performed after extraction, and the sockets were not sutured. In the immediate postoperative period, analgesia was provided by the residual effect of ketamine hydrochloride; boosted tramadol $(50 \mathrm{mg} / \mathrm{mL}$; Tramal 50, Pfizer, Guarulhos, São Paulo, Brazil) was injected intraperitoneally (0.025 mL/100 g weight) for analgesia. Then, for 3 consecutive days, acetaminophen drops $(200 \mathrm{mg} / \mathrm{mL})$ were added to the animal's drinking water $(5 \mathrm{mg} / \mathrm{mL}$ of water; Tylenol, JannsenCilag Farmacêutica Ltda, São José dos Campos, São Paulo, Brazil). In addition, the chow was crushed and mixed with water during the first 3 postoperative days to facilitate eating. After these procedures, animals remained in their plastic boxes. During the postanesthesia recovery period, warm compresses were placed on the bedding until animals were fully awake. As noted above, all animals were kept under controlled temperature $\left(24^{\circ} \mathrm{C} \pm 2{ }^{\circ} \mathrm{C}\right)$, humidity, and ventilation conditions, in a 12-h light-dark cycle, throughout the experiment.

\subsection{Low-Level Laser Therapy Protocol}

Laser therapy was administered using an aluminum-galliumarsenide (AlGaAs) diode laser system (Thera Lase/DMC Equipamentos, São Paulo, Brazil). Of the two experimental groups, one received irradiation in the red range (685-nm wavelength) at $0.3 \mathrm{~W}$ power. Animals in the other group received irradiation in the infrared range (830-nm wavelength) at $0.9 \mathrm{~W}$ power. In both groups, energy was set to $4 \mathrm{~J}$. The energy density was $67 \mathrm{~J} / \mathrm{cm}^{2}$ and the power density was $5 \mathrm{~W} / \mathrm{cm}^{2}$ (duration of $134 \mathrm{~s}$ per spot) for the red laser. The energy density was $67.5 \mathrm{~J} / \mathrm{cm}^{2}$ and $15 \mathrm{~W} / \mathrm{cm}^{2}$ (duration of $45 \mathrm{~s}$ per spot) for the infrared laser. Irradiation was performed in continuous wave mode, with a spot size (emission area) of $0.06 \mathrm{~cm}^{2}$. All spot applications were performed intraorally, in contact with the 


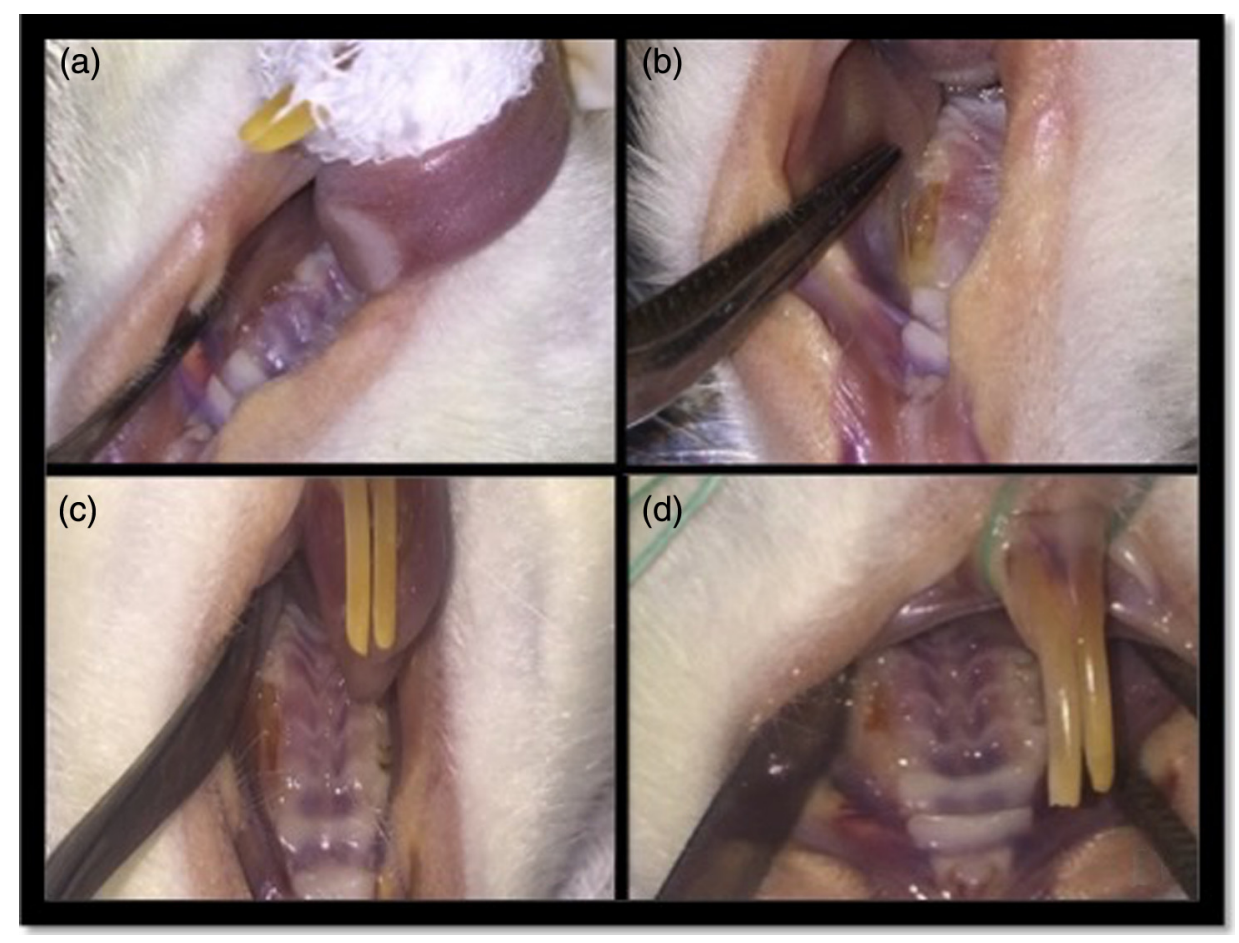

Fig. 1 Clinical appearance of healing 15 days after extraction of left upper molars. (a) Group 1 (vehicle control), MD dimension $=0 \mathrm{~mm}$; (b) group 2 (medication control), MD dimension $=6 \mathrm{~mm}$; (C) group 3 (R laser), $M D$ dimension $=2 \mathrm{~mm}$; and (d) group 4 (IR laser), $M D$ dimension $=1 \mathrm{~mm}$.

central region of the surgical wound, both during the intraoperative period and every $24 \mathrm{~h}$ for 15 days thereafter.

\subsection{Humane Endpoints}

Pain and distress were minimized during all procedures involved in this study. In case of distress, animals would be euthanized by sedation followed by deep anesthesia.

\subsection{Euthanasia}

At the end of the experimental period, animals were randomly selected and euthanized by deep anesthesia with isoflurane, until loss of all reflexes and rigor mortis were confirmed.

\subsection{Clinical and Histological Examination}

All specimens from all groups were examined grossly for progression of the alveolar repair process. A detailed descriptive analysis of clinical findings was performed. A digital camera was used to obtain photographs of the surgical sites for later analysis by the same blinded observer (R1 and R2), as well as by a second observer $(\mathrm{J})$. Interobserver agreement regarding mucosal healing was evaluated by the kappa statistic ( $1=$ unsatisfactory, 2 = satisfactory, and $3=$ highly satisfactory). In addition, the mesiodistal (MD) dimension of the surgical wound was measured with a graduated probe.

Histological analysis was performed by an oral pathologist and a calibrated examiner, both of whom were blinded. At least four fields from each specimen were analyzed under $100 \times$ magnification, and a descriptive, qualitative analysis was performed for bone changes, vascularization, inflammatory infiltrate, osteoblast activity, presence of sequestered or necrotic bone, viable osteocytes, newly formed bone, and epithelial tissue integrity. Then, the kappa statistic was used for analysis of interobserver agreement between the oral pathologist and the second examiner, who had been calibrated by the pathologist. Vascularity was assessed on a four-point scale $(0=$ absent, $1=$ slight $; 2=$ moderate, $3=$ marked), as was inflammatory infiltration $[\mathrm{A}=$ no cells, $\mathrm{B}=1$ to 100 cells (slight), $\mathrm{C}=100$ to 250 cells (moderate), and $\mathrm{D} \geq 250$ cells (severe)]. These data were entered into a table for statistical analysis.

\subsection{Statistical Analysis}

Data were entered into Microsoft Excel 2010 and exported to PASW Statistics Version 18.0 for statistical analysis. Categorical variables were described as absolute and relative frequencies and quantitative variables, such as medians and ranges. Fisher's exact test was used to associate these with categorical variables. Outliers were detected via adjusted residuals analysis. Quantitative variables were compared by the Kruskal-Wallis test. For multiple comparisons, variables were ranked and analyzed using Tukey's post hoc test. As noted above, the kappa coefficient was used to assess intra- and interexaminer agreement. Kappa $(\kappa)$ values $>0.75$ represent excellent agreement, those $<0.40$ represent low agreement, and those in the 0.40 to 0.75 range represent moderate agreement. The significance level was set at $5 \%$.

\section{Results}

Overall, the animals tolerated the procedure well, achieved good hemostasis, and recovered well from anesthesia. Seven animals died during the experiment. Therefore, 21 rats were included in the study, distributed as follows: vehicle control group, $n=6$; medication control group, $n=5$; red laser group, $n=5$; and infrared laser group, $n=5$. 
Table 1 Table comparing measurement mesiodistal (MD) and vestibular palatine (VP) dimension of the surgical wounds between groups.

\begin{tabular}{lccccc} 
& Control $n=6$ & ZA control $n=5$ & IR laser $n=5$ & Red laser $n=5$ & $p$ \\
\hline MD wound dimension & $2.3(0 \text { to } 3)^{\mathrm{a}}$ & $5(3 \text { to } 6)^{\mathrm{b}}$ & $4(1 \text { to } 6)^{\mathrm{a}, \mathrm{b}}$ & $3.5(2 \text { to } 5)^{\mathrm{a}, \mathrm{b}}$ & $\mathbf{0 . 0 3 5}$ \\
VP wound dimension & $0.8(0 \text { to } 1)^{\mathrm{a}}$ & $1.5(1 \text { to } 4)^{\mathrm{a}, \mathrm{b}}$ & $2(0.5 \text { to } 3.5)^{\mathrm{a}, \mathrm{b}}$ & $2(1 \text { to } 4)^{\mathrm{b}}$ & $\mathbf{0 . 0 4 2}$ \\
\hline
\end{tabular}

Note: Data shown as median (range) and compared by Kruskal-Wallis test. Different superscript letters denote significant difference between groups.

IR, infrared; MD, mesiodistal; VP, vestibulopalatal; and ZA, zoledronic acid.

The bold value shown that the measurements wound dimensions. Considering $p<0,001$.

On clinical evaluation, specimens from group 1 (vehicle control) exhibited highly satisfactory healing. In group 4 (IR laser), healing was satisfactory. Wounds were not entirely covered by mucosa, but granulation tissue was present, with no signs of infection or inflammation and no exposed bone (Fig. 1). The best outcomes in terms of median MD dimension were obtained in group 1 (vehicle control), as shown in Table 1 and Fig. 2. Healing in groups 2 (medication control) and 3 (R laser) ranged from unsatisfactory to satisfactory. The difference between these groups was attributed to the state of wounds in group 2 (medication control), which exhibited erythematous, edematous margins, exposed bone in some specimens, and larger median MD dimensions. However, there were no appreciable signs of infection in any specimen from any group. Regarding

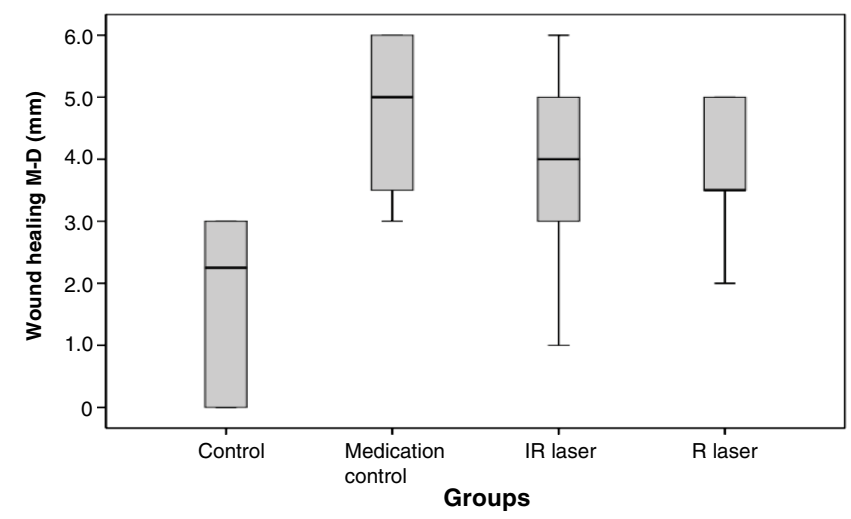

Fig. 2 Comparative boxplot graph of the MD wound healing between groups. assessment of healing, there were no significant differences across groups. Kappa coefficients were consistent with moderate intrarater agreement $(\kappa=0.64, p<0.001)$. Interrater agreement in assessment of healing was moderate $(\kappa=0.57, p<0.001)$ in one situation and excellent $(\kappa=0.78, p<0.001)$ in another. Regarding MD dimension, measurements were significantly smaller in group 1 (vehicle control) than in group 2 (medication control) (Table 1 and Fig. 2).

On histological analysis, specimens from groups 1 (vehicle control) and 2 were consistent with marked vascularization in three animals each, representing $50 \%$ and $60 \%$ of animals, respectively (Fig. 3). Specimens from groups 3 (R laser) and 4 (IR laser) were similar in terms of vascularization, which was moderate in 4 of 5 samples from each group (80.0\%). The intensity of inflammatory cell infiltration (ICI) in groups 1 (vehicle control) and 2 (medication control) largely ranged from moderate $(50 \%)$ to severe $(40 \%)$. In most specimens from groups 3 (R laser) and 4 (IR laser), ICI was slight (60\% and $40 \%$, respectively) or moderate (40\% of specimens from each group). Despite the aforementioned differences in clinical and histological parameters, no statistically significant differences in vascularity or ICI were detected by any examiner among the study groups as shown in Table 2 . Interrater agreement was excellent for assessment of vascularity $(\kappa=0.91, p<0.001)$ and ICI $(\kappa=0.92, p<0.001)$. Table 3 summarizes these results. The topographic distribution of inflammatory infiltrates was similar across all groups (near bone sequestra, root remnants, and tooth crowns). Groups 2 (medication control), 3 (R laser), and 4 (IR laser) exhibited areas of bone necrosis (e.g., in the alveolar ridge) in addition to sequestra, and ICI was observed in these regions as well. In groups 2 (medication control), 3 (R laser), and 4 (IR laser), areas of bone necrosis with

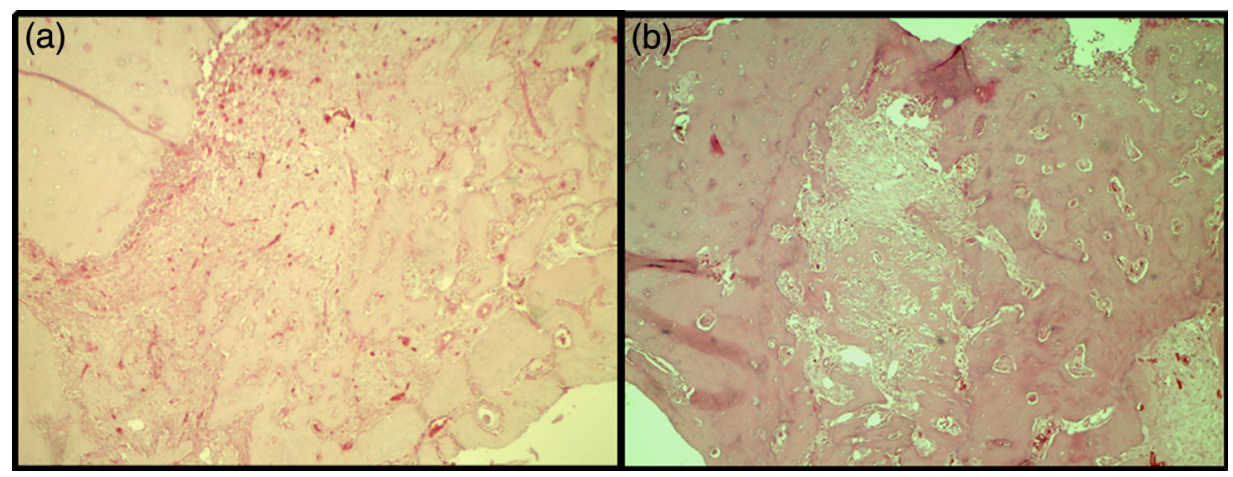

Fig. 3 (a) Histological appearance (100x magnification) of a slice of alveolar bone from a group 2 (medication control) specimen, showing granulation tissue filling the alveolar socket and an exposed bone spicula on the surface. (b) Detail showing the alveolar ridges contain empty lacunae devoid of viable osteocytes, which denotes bone necrosis. 
Table 2 Table comparing the groups in relation to the microscopic assessment performed by two observers.

\begin{tabular}{|c|c|c|c|c|c|}
\hline & $\begin{array}{l}\text { Vehicle control } \\
\quad n=6\end{array}$ & $\begin{array}{l}\text { Medication control } \\
\quad n=5\end{array}$ & $\begin{array}{l}\text { Laser IR } \\
n=5\end{array}$ & $\begin{array}{c}\text { Laser R } \\
n=5\end{array}$ & $P$ \\
\hline Vascularization $\mathrm{R}$ & & & & & 0.472 \\
\hline Slight & $1(16.7)$ & - & & & \\
\hline Moderate & $2(33.3)$ & $2(40.0)$ & $4(80.0)$ & $4(80.0)$ & \\
\hline Marked & $3(50.0)$ & $3(60.0)$ & $1(20.0)$ & $1(20.0)$ & \\
\hline Vascularization $\mathrm{F}$ & & & & & 0.472 \\
\hline Slight & $1(16.7)$ & - & - & - & \\
\hline Moderate & $2(33.3)$ & $2(40.0)$ & $4(80.0)$ & $4(80.0)$ & \\
\hline Marked & $3(50.0)$ & $3(60.0)$ & $1(20.0)$ & $1(20.0)$ & \\
\hline $\mathrm{ICI} R$ & & & & & 0.438 \\
\hline Slight ( 1 to 100 cells): sparse mononuclear cells & - & $1(20.0)$ & $2(40.0)$ & $3(60.0)$ & \\
\hline $\begin{array}{l}\text { Moderate ( } 100 \text { to } 250 \text { cells): mononuclear infiltrate } \\
\text { and/or sparse neutrophils }\end{array}$ & $3(50.0)$ & $2(40.0)$ & $2(40.0)$ & $2(40.0)$ & \\
\hline Severe ( $>250$ cells): polymorphonuclear neutrophil infiltrate & $3(50.0)$ & $2(40.0)$ & $1(20.0)$ & - & \\
\hline $\mathrm{ICI} F$ & & & & & 0.178 \\
\hline Slight ( 1 to 100 cells): sparse mononuclear cells & - & $1(20.0)$ & - & $3(60.0)$ & \\
\hline $\begin{array}{l}\text { Moderate ( } 100 \text { to } 250 \text { cells): mononuclear infiltrate } \\
\text { and/or sparse neutrophils }\end{array}$ & $3(50.0)$ & $2(40.0)$ & $4(80.0)$ & $2(40.0)$ & \\
\hline Severe (>250 cells): polymorphonuclear neutrophil infiltrate & $3(50.0)$ & $2(40.0)$ & $1(20.0)$ & - & \\
\hline
\end{tabular}

Note: Data presented by $n(\%)$ and compared by Fisher's exact test.

osteocyte loss were observed in the bone matrix of the alveolar ridge, although a smaller number of these areas occurred in groups 3 ( $\mathrm{R}$ laser) and 4 (IR laser) when compared to the group 2 (medication control), which also exhibited alveolar wall necrosis in some specimens (Fig. 4). In group 1 (vehicle control) and in some samples from groups 3 (R laser) and 4 (IR laser), there was evidence of sequestrum resorption, with Howship's lacunae and portions excavated by osteoclast activity (Fig. 5).

Groups 1 (medication control), 3 (R laser), and 4 (IR laser) exhibited similar new bone formation, with trabeculae forming septae in vascularized primary bone, osteoblast activity, and bone-marrow cells (Fig. 6). In some specimens from these three groups, there was evidence of a consolidated haversian system, with concentric lamellae surrounding a haversian canal containing blood vessels. Newly formed bone exhibited centrifugal growth from the bottom to the midpoint of the alveolar socket. From the midpoint to the surface, the alveolar socket was filled with granulation tissue, which was more mature than in group 2 (medication control) animals, composed predominantly of fibroblasts. In group 2 (medication control), granulation tissue was observed to be at earlier stages of maturation, with relatively little new bone formation in the alveolar floor, walls, and region of the alveolar septum. Due to the presence of bone spicules and tooth remnants, there was no evidence of established epithelium covering the wound surface completely in any group, but there was formation of epithelial tissue. It is important to note that, on histological examination, in specimens from groups 3 and 4, the alveolar sockets were covered

Table 3 Table presentation of some results achieved in the study.

\begin{tabular}{lcccc}
\hline & Mucosal healing & MD dimension surgical wound $(\mathrm{mm})$ & Vascularization & Inflammatory infiltrate \\
\hline Group 1 (vehicle control) & highly satisfactory & 2.3 & Marked & Severe/moderate \\
Group 2 (medication control) & unsatisfactory & 5 & Marked & Severe/moderate \\
Group 3 (R laser) & satisfactory & 3.5 & Moderate & Slight \\
Group 4 (IR laser) & satisfactory & 4 & Moderate & Moderate \\
\hline
\end{tabular}




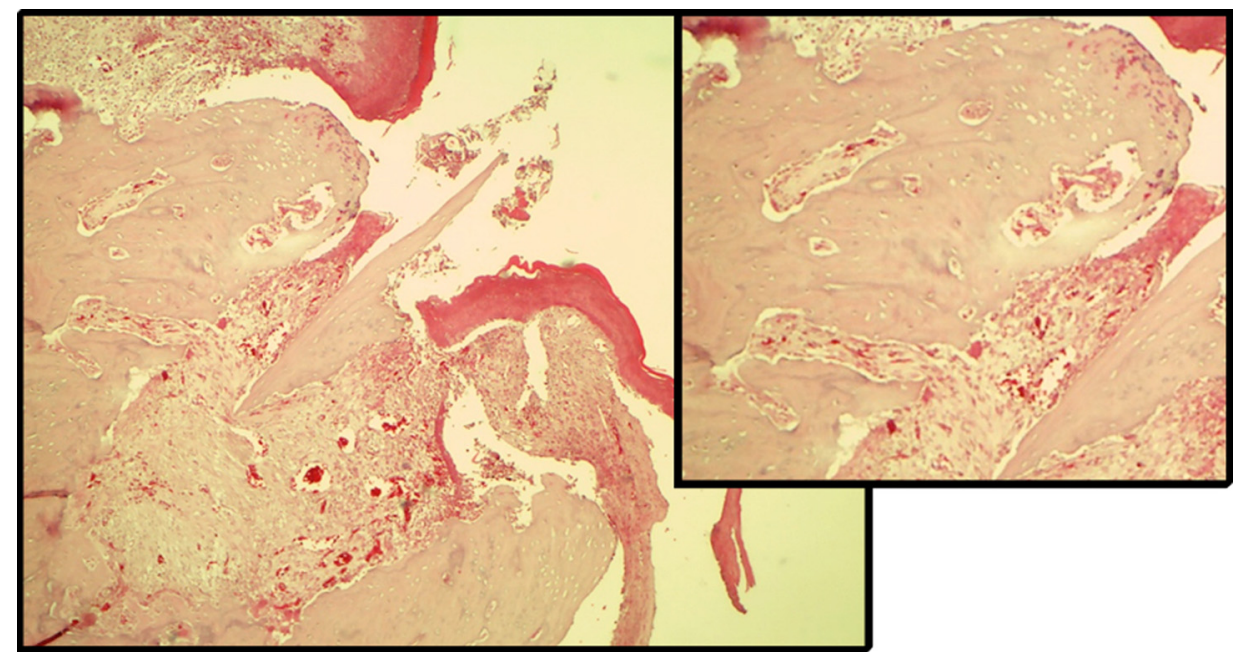

Fig. 4 Histological appearance (100x magnification) of a slice of alveolar bone from a group 2 (medication control) specimen, showing granulation tissue filling the alveolar socket and an exposed bone spicula on the surface. Inset, upper left: detail showing the alveolar ridges contain empty lacunae devoid of viable osteocytes, which denotes bone necrosis.

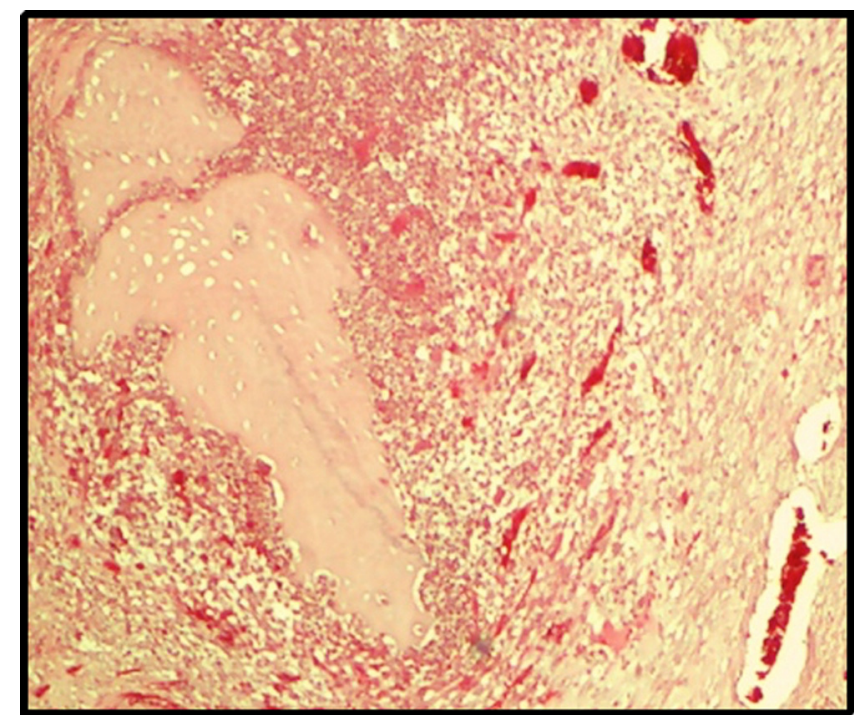

Fig. 5 Histological appearance (100x magnification) of a bone sequestrum surrounded by marked $\mathrm{ICl}$ in a group 1 (vehicle control) specimen. There is evidence of osteoclast activity, including Howship's lacunae (excavations in bone tissue caused by resorption of the outer surface of the sequestered bone by osteoclasts). by granulation tissue, although epithelial coverage was incomplete. In one specimen from group 4 (IR laser), a substantial portion of whole epithelial tissue was visualized (Fig. 7).

Final body weight was greater in group 1 (vehicle control) than in the other groups, across which this parameter was similar. This difference in weight was significantly greater in group 1 (vehicle control) than in the other study groups. These values are shown in Table 4.

\section{Discussion}

The objective of this study was to assess the stimulating effects of red- and infrared-spectrum LLLT on bone and soft-tissue repair in rats exposed to ZA and DX. The protocol of ZA used in this experiment was sufficient to induce changes at the surgical site as compared to controls not treated with BPs. These changes in healing were manifested clinically as mucosal erythema and edema, bone exposure, and a marked, statistically significant difference in body weight between the vehicle control group and all other groups. The findings of this study are consistent with established criteria for diagnosis of BRONJ. Few studies of LLLT have been conducted in animals, particularly in the maxilla. Therefore, on the basis of some existing studies, we defined LLLT protocols using two distinct wavelengths. Experimental studies have demonstrated the photostimulatory

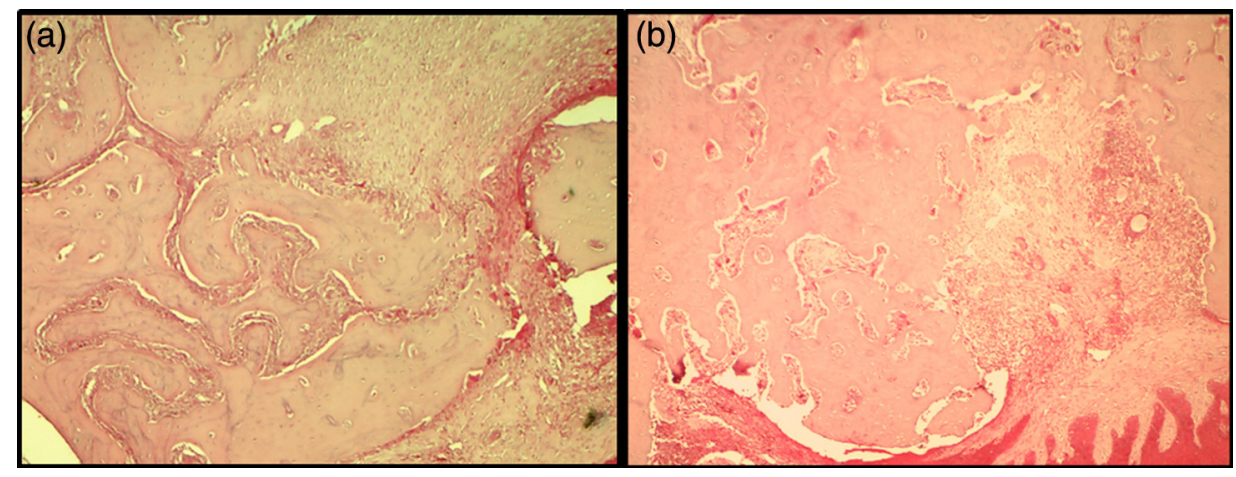

Fig. 6 Histological appearance (100× magnification) of (a) a group 1 (vehicle control) specimen and (b) a group 3 ( $R$ laser) specimen. There is evidence of healing, with similar tissue organization and new bone formation, including trabeculae in vascularized primary bone, osteoblast activity, and bone-marrow cells. 


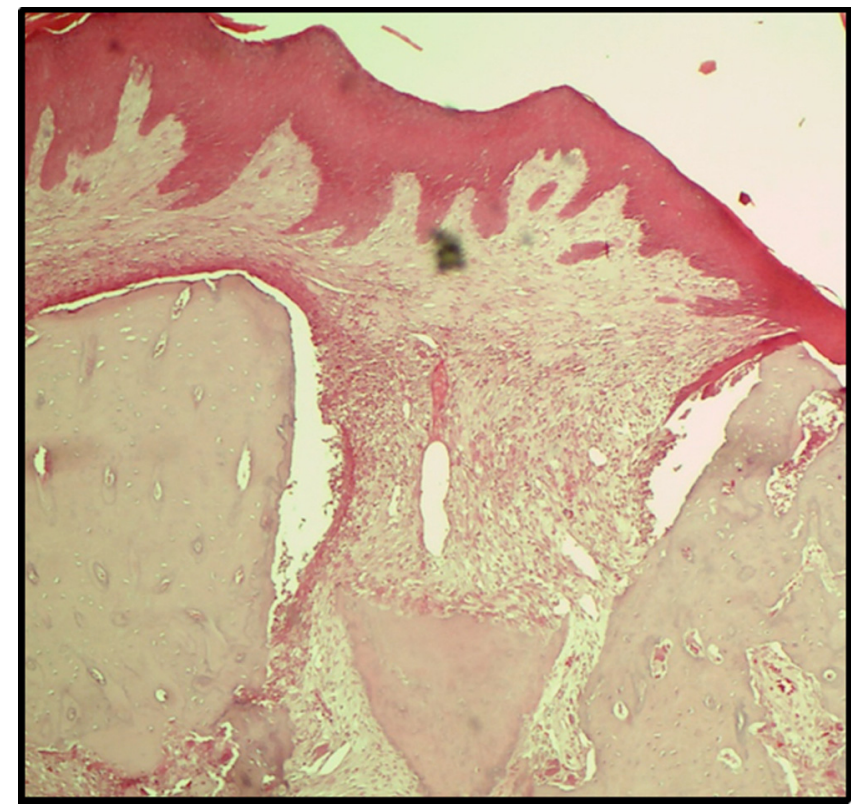

Fig. 7 Histological appearance (100x magnification) of a group 4 (IR laser) specimen, showing substantial epithelial tissue integrity.

effects of LLLT; combined with BP therapy or otherwise, it has been used effectively to stimulate bone formation in artificially created cavities $\left(830 \mathrm{~nm}, 50 \mathrm{~mW}, 4 \mathrm{~J} / \mathrm{cm}^{2}\right)$, in surgical fractures in rats $\left(904 \mathrm{~nm}, 4 \mathrm{~mW} \mathrm{~cm}^{-2}, 0.72 \mathrm{~J} \mathrm{~cm}^{-2}\right),{ }^{17,21}$ and to boost soft-tissue wound healing $\left(660 \mathrm{~nm}, 100 \mathrm{~mW}, 10 \mathrm{~J} / \mathrm{cm}^{2}\right){ }^{22}$

The histological analyses of our study are largely descriptive and static. The individual phases (inflammation, vascularity, bone healing) occur over various time frames, so this can be a significant limitation of this study. Although there were no statistically significant differences in vascularization or ICI in our sample, histological analysis revealed a clear difference in tissue healing maturation stage between the LLLT groups and the other groups, including group 1 (vehicle control). In groups 3 ( $\mathrm{R}$ laser) and 4 (IR laser), there was moderate vascularization, which may be interpreted as a result of faster maturation of granulation tissue (which promoted faster healing) after 2 weeks of LLLT irradiation. Similar results have already been reported. ${ }^{22}$ In group 1 (vehicle control) and in both laser groups, new bone formation was found predominantly in the floor and middle of the alveolar sockets, and, in some specimens, sockets were completely filled with bone or organized granulation tissue. These results are similar to those reported by Freitas et al., ${ }^{20}$ who also found new bone formation in surgically fractured tibias irradiated daily with a HeNe laser (energy density $94.7 \mathrm{~J} / \mathrm{cm}^{2}$ ) for 14 days. The treated group exhibited faster, progressive bone consolidation as compared to controls. ${ }^{20}$ In this study, some specimens of group 4 (IR laser) exhibited evidence of healing as satisfactory and in group 1 (vehicle control) highly satisfactory. Furthermore, although the epithelium was not completely closed, one sample had a substantial portion of epithelial tissue with preserved integrity. Surgical sites were not sutured in this study; i.e., all healing occurred by secondary intention. This is an important finding because the epithelial barrier prevents the development of infection, which is one of the most common wound complications. ${ }^{22}$ Another difference between group 1 (vehicle control) and the other groups concerns bone necrosis, which was observed in the alveolar ridge in groups 2 (medication control) and, to a lesser extent, in groups 3 (R laser) and 4 (IR laser). This difference may be justified by the actions of BPs on bone tissue, which would have predisposed animals in group 2 (medication control) to a larger open wound area (which constitutes a worse outcome in relation to the other groups). Results similar to ours have been reported in studies using the same laser system, even with lower doses and higher power settings. In one of this studies, the authors used a 5-day course of daily LLLT $\left(660 \mathrm{~nm}, 100 \mathrm{~mW}, 10 \mathrm{~J} / \mathrm{cm}^{2}, 20 \mathrm{~s} / \mathrm{spot}, 2 \mathrm{~J} / \mathrm{spot}\right.$ ) for traumatic wounds of the dorsal region in rats. ${ }^{22}$ Another study reported increased trabecular volume in the lumbar and thoracic vertebrae of rats treated with a BP (alendronate) and LLLT $(830 \mathrm{~nm}$, $\left.50 \mathrm{~mW}, 4 \mathrm{~J} / \mathrm{cm}^{2}, 30 \mathrm{~s} / \mathrm{spot}, 1.5 \mathrm{~J} / \mathrm{spot}\right){ }^{17}$ This positive effect of the combination of laser and BP therapy may be related by an increase in osteoblast number and activity, with trabeculae formed by more than one row of osteoblasts and massive amounts of osteoid. ${ }^{17}$

Furthermore, the stimulant effect of the laser occurs during the early stages of cell proliferation and differentiation and does not take place at later stages. ${ }^{23}$ The photobiomodulatory effect of the laser is not always detectable, as its magnitude will depend on the physiological status of the cell at the time of irradiation. ${ }^{19,24}$ Several laser therapy protocols for stimulation of bone metabolic activity have been designed, with differences ranging from laser type and dose to the method of irradiation, which hinders comparison of results across studies. LLLT is dependent on many factors, including wavelength, dose, energy density, duration of irradiation, treatment frequency, lesion type, and target tissue.

\section{Conclusions}

We conclude that, under the conditions used in this study, combined administration of $\mathrm{ZA}$ and DX induces osteonecrosis of

Table 4 Table comparing body weight at baseline, in the day of euthanasia and the difference in body weight between groups.

\begin{tabular}{lccccc} 
& Control $n=6$ & ZA control $n=5$ & IR laser $n=5$ & Red laser $n=5$ & $p$ \\
\hline Body weight at baseline & $252.5(250$ to 275$)$ & $255(250$ to 275$)$ & $250(240$ to 275$)$ & $250(250$ to 275$)$ & 0.553 \\
Body weight euthanasia & $400(350 \text { to } 425)^{\mathrm{a}}$ & $300(250 \text { to } 300)^{\mathrm{b}}$ & $270(230 \text { to } 300)^{\mathrm{b}}$ & $290(240 \text { to } 300)^{\mathrm{b}}$ & $\mathbf{0 . 0 0 4}$ \\
Difference in body weight & $100(65 \text { to } 150)^{\mathrm{a}}$ & $40(-25 \text { to } 50)^{\mathrm{b}}$ & $20(-20 \text { to } 50)^{\mathrm{b}}$ & $40\left(-10{\text { to } 50)^{\mathrm{b}}}^{\mathbf{0 . 0 1 0}}\right.$ \\
\hline
\end{tabular}

Note: Data shown as median (range) and compared by Kruskal-Wallis test. Different superscript letters denote significant difference between groups. IR, infrared; MD, mesiodistal; VP, vestibulopalatal; ZA, zoledronic acid.

The bold values shown that the body weight in the day of euthanasia and the difference in weight at baseline were significantly greater in group 1 (vehicle control) than in the other study groups. Considering $p<0,001$. 
the jaw and delays healing in rats. The LLLT with an AlGaAs diode in the red $(685 \mathrm{~nm})$ and infrared $(830 \mathrm{~nm})$ ranges had a positive influence on the bone and soft-tissue repair process with no significant difference between them. However, further studies are needed to confirm its potential for use in cases related to BP therapy.

\section{Disclosures}

The authors have no competing interests to declare and no conflicts of interest.

\section{Acknowledgments}

We thank Coordination for the Improvement of Higher Education Personnel (CAPES) for the Program for Academic Excellence (PROEX) grant awarded; all staff at PUCRS Building14 (Center for Experimental Biological Models); the faculty and staff at the Pathology Laboratory, PUCRS School of Dentistry; and nurse Sandra and staff pharmacists Fernanda, Natalia, Carolina, and Patrícia at CliniOnco, Porto Alegre, RS, Brazil.

\section{References}

1. M. Luomanen and S. Alaluusua, "Treatment of bisphosphonate-induced osteonecrosis of the jaws with Nd:YAG laser biostimulation," Lasers Med. Sci. 27(1), 251-255 (2012).

2. M. G. Guarda et al., "Laser GaAlAs $(860 \mathrm{~nm})$ photobiomodulation for the treatment of bisphosphonate-induced osteonecrosis of the jaw," Photomed. Laser Surg. 30(5), 293-297 (2012).

3. A. Gegler et al., "Bisfosfonatos e osteonecrose maxilar: revisão da literatura e relato de dois casos," Rev. Bras. Cancerol. 52(1), 25-31 (2006).

4. M. T. Drake, B. L. Clarke, and S. Khosla, "Bisphosphonates: mechanism of action and role in clinical practice," Mayo Clin. Proc. 83(9), 1032-1045 (2008).

5. B. Kan et al., "Low-level laser therapy supported teeth extractions of two patients receiving IV zolendronate," Lasers Med. Sci. 26(5), 569-575 (2011)

6. B. W. Nevile et al., "Lesões físicas e químicas," in Patologia Oral e Maxilofacial, B. W. Nevile et al., Eds., pp. 287-330, 3rd Ed., Elsevier, São Paulo (2009).

7. J. E. Dunford et al., "Structure-activity relationships for inhibition of farnesyl diphosphate synthase in vitro and inhibition of bone resorption in vivo by nitrogen-containing bisphosphonates," J. Pharm. Exp. Ther. 296(2), 235-242 (2001).

8. F. Angiero et al., "Osteonecrosis of the jaws caused by bisphosphonates: evaluation of a new therapeutic approach using the Er:YAG laser," Lasers Med. Sci. 24(6), 849-856 (2009).
9. M. Scoletta et al., "Effect of low-level laser irradiation on bisphosphonate-induced osteonecrosis of the jaws: preliminary results of a prospective study," Photomed. Laser Surg. 28(2), 179-184 (2010).

10. P. Vescovi et al., "Nd:YAG laser biostimulation of bisphosphonateassociated necrosis of the jawbone with and without surgical treatment," Br. J. Oral Maxillofac. Surg. 45(8), 628-632 (2007).

11. S. L. Ruggiero et al., "Osteonecrosis of the jaws associated with the use of bisphosphonates: a review of 63 cases," J. Oral Maxillofac. Surg. 62(5), 527-534 (2004).

12. S. L. Ruggiero et al., "American Association of Oral and Maxillofacial Surgeons position paper on bisphosphonate-related osteonecrosis of the jaws-2009 update," J. Oral Maxillofac. Surg. 67(1), A2 (2009).

13. P. Vescovi et al., "Nd:YAG Biostimulation in the treatment of bisfosfonate associated osteonecrosis of the jaw: clinical experiences 28 cases," Photomed. Laser Surg. 26(1), 37-46 (2008).

14. S. B. Woo, J. W. Hellstein, and J. R. Kalmar, "Systematic review: bisphosphonates and osteonecrosis of the jaws," Ann. Intern. Med. 144(10), 753-761 (2006).

15. V. Fusco et al., "Decreasing frequency of osteonecrosis of the jaw in cancer and myeloma patients treated with bisphosphonates: the experience of the oncology network of piedmont and aosta valley (NorthWestern Italy)," ISRN Oncol. 2013, 1-8 (2013).

16. S. L. Ruggiero et al., "American association of oral and maxillofacial surgeons position paper on medication-related osteonecrosis of the jaw-2014 update," J. Oral Maxillofac. Surg. 72(10), 1938-1956 (2014).

17. J. S. Diniz et al., "Effect of low-power gallium-aluminum-arsenium laser therapy $(830 \mathrm{~nm})$ in combination with bisphosphonate treatment on osteopenic bone structure: an experimental animal study," Lasers Med. Sci. 24(3), 347-352 (2009).

18. S. T. Sonis et al., "Bony changes in the jaws of rats treated with zoledronic acid and dexamethasone before dental extractions mimic bisphosphonate-related osteonecrosis in cancer patients," Oral Oncol. 45(2), 164-172 (2009).

19. V. G. Garcia et al., "Effects of LLLT in combination with bisphosphonate on bone healing in critical size defects: a histological and histometric study in rat calvaria," Lasers Med. Sci. 28(2), 407-414 (2013).

20. I. G. F. Freitas, V. Baranauskas, and M. A. Cruz-Höfling, "Laser effects on osteogenesis," Appl. Surf. Sci. 154, 548-554 (2000).

21. J. Nissan et al., "Effect of low intensity laser irradiation on surgically created bony defects in rats," J. Oral Rehabil. 33(8), 619-924 (2006).

22. F. C. F. S. Calisto et al., "Use of low-power laser to assist the healing of traumatic wounds in rats," Acta Cir. Bras. 30(3), 204-208 (2015).

23. Y. Ozawa et al., "Low-energy laser irradiation stimulates bone nodule formation at early stages of cell culture in rat calvarial cells," Bone 22(4), 347-354 (1998).

24. T. Karu, L. Pyatibrat, and G. Kalendo, "Irradiation with He-Ne laser increases ATP level in cells cultivated in vitro," J. Photochem. Photobiol. 27(3), 219-223 (1995).

Biographies for the authors are not available. 